\title{
Emotional Semantic Analysis of Male T-shirt Image Based on Color and Texture Features
}

\author{
Xiaomeng Zhanag ${ }^{1, ~ a, ~ H a i b o ~ Z h a n g, ~ b, ~ * ~}$ \\ ${ }^{1}$ Information Center, Beijing Institute of Fashion Technology, Beijing, 100029, China \\ ${ }^{2}$ Library, Beijing Institute of Fashion Technology, Beijing, 100029, China \\ a2540944916@qq.com, bhbdmzhb@126.com, *corresponding author
}

Keywords: male T-shirt image; low-level feature extraction; emotional semantic; color feature; texture feature

\begin{abstract}
In order to recognize and retrieve the emotional semantics of male T-shirt images, it is necessary to extract the low-level features of the color and texture of Images and analyze the emotional semantics to find out the relationship between the image features and emotional semantics. To study the male T-shirt image emotional description and establish the three-dimensional emotional factor space, on the basis of this, the corresponding relationships among color, texture features and three factors of T-shirt images are analyzed. The results show that the first factor can be explained by 10-dimensional features of saturation-cold and warm fuzzy histogram plus 1-dimentional contrast, the second factor can be represented a feature of 256-dimentional gray scales plus 1-dimentional color contrast, the third factor can be used 3-dimentional Tamura features (coarseness, contrast, directionality)to characterize. This relationship lays the foundation for achieving emotional semantic recognition and retrieval of content-based T-shirt images.
\end{abstract}

\section{Introduction}

Most of the external information that humans perceive is obtained through one of the important ways for humans to obtain information of vision. So, humans can perceive not only the world of this entity, but also the semantics of emotion. With the development of computer and information technology, how to let the computer feel the emotion brought by the clothing image through visual perspective, and identify and retrieve the emotional semantics of the clothing image, has received more and more attention in the industry. The research results have been applied in clothing, art design, and apparel e-commerce.

The image of male T-shirt can give a certain amount of emotion, and different feelings are related to their essential attributes such as color, material, texture, texture and so on. This paper specifically analyzes the emotional semantics of male T-shirt images, trying to find the relationship between image features and emotional semantics.

At present, there is not much emotional semantic analysis for clothing images. The literature [1] analyzes the relationship between low-level fabric features and emotional semantic space; the same literature [2] analyzes the relationship between low-level features of male suits and emotional semantic space; literature [3] takes the image features as an example to realize the emotional semantic classification and retrieval of art images. There are few researches on emotional semantic recognition and retrieval of content-based male T-shirt images. Based on the previous research, this paper the low-level features of the image closely related to the male T-shirt factor 1, factor 2 and factor 3 are analyzed, and the relationship between the low-level features and the affective factors is established, which lays a foundation for the emotional semantic recognition and retrieval of male T-shirt images. 


\section{Male T-shirt Image Emotion Factor Space Model}

In the early stage, to investigate the male T-shirt image sentiment words on line, and to take the expert opinions in the field of clothing. Finally, 8 pairs of anti-sense emotional words were determined, and the subjects were subjectively scored online. The eight pairs of emotional words are "simple - gorgeous", "calm - sportive", "formal - casual", "plain - fashionable", "classical-modern", "elegant - rugged", "gentle - strong", "implicit - flamboyant". Then, through a multivariate statistical factor analysis method, the relationship between emotional words and emotional factors is analyzed and a spatial model of emotional factors for male T-shirt images is established. The factor analysis is used to attribute the 8-dimensional emotional semantics into three independent factors, and the relationship model between emotional semantics and three factors is established:

Simple - Gorgeous $=0.945 f_{1}-0.056 f_{2}+0.078 f_{3}$

Calm - Sportive $=0.203 f_{1}+0.922 f_{2}+0.152 f_{3}$

Formal - Casual $=0.111 f_{1}+0.962 f_{2}+0.108 f_{3}$

Plain - Fashionable $=0.914 f_{1}+0.104 f_{2}-0.189 f_{3}$

$$
\text { Classical - Modern }=0.774 f_{1}+0.494 f_{2}-0.150 f_{3}
$$

Elegant - Rugged $=0.159 f_{1}+0.466 f_{2}+0.823 f_{3}$

Gentle - Strong $=-0.247 f_{1}-0.047 f_{2}+0.922 f_{3}$

Implicit - Flamboyant $=0.829 f_{1}+0.455 f_{2}+0.006 f_{3}$

In the equation (1) relational model, $f_{1}, f_{2}$ and $f_{3}$ are three factors derived from factor analysis of the Scree plot. Three factors can account for $90.524 \%(>85 \%)$ of the total variance of the 8-dimensional emotional semantics. According to factor analysis and principal component analysis, the factor load matrix is obtained by orthogonal rotation of the maximum variance method, then the factor score coefficient is estimated by regression method to make factor score function [4].

$f_{1}=0.413 \times($ simple - gorgeous $)-103 \times($ calm - sportive $)-0.156 \times($ formal - casual $)+0.328 \times($ plain - fashionable $)+0.188 \times($ classical - modern $)+0.072 \times($ elegant - rugged $)+0.033 \times($ gentle - strong $)$ $+0.240 \times$ (implicit - flamboyant)

$f_{2}=-0.259 \times($ simple - gorgeous $)+0.435 \times($ calm - sportive $)+0.487 \times($ formal - casual $)-0.109 \times$ (plain-fashionable) $+0.130 \times($ classical-modern $)+0.055 \times($ elegant-rugged $)-0.158 \times($ gentle - strong $)$ $+0.065 \times$ (implicit - flamboyant)

$f_{3}=0.193 \times($ simple - gorgeous $)-0.054 \times($ calm - sportive $)-0.106 \times($ formal - casual $)-0.030 \times$ (plain - fashionable) $-0.101 \times($ classical - modern $)+0.501 \times($ elegant - rugged $)+0.620 \times($ gentle strong) $+0.023 \times$ (implicit - flamboyant) $(2)$

According to formula (2), the three emotion factors of each image can be calculated from the corresponding emotion descriptor value and factor score coefficient to explore and analyze the emotional semantics of the male T-shirt image.

Each factor corresponds to a different pair of emotional words, some of which have a larger proportion. For example, factor 1 and "simple - gorgeous", "plain - fashionable" emotional words are more relevant; factor 2 is related to "formal - casual", "calm - sportive" emotional words; factors 3 is related to the emotional words of "gentle - strong" and "elegant - rugged". Therefore, in order to automatically calculate the emotional factor value of the image, the essential features of the emotional word pair of the image are closely related to the three factors.

According to color psychology, in terms of the color characteristics of human vision, the three elements of brightness, saturation, and warm and cold tones mainly constitute color. 


\section{Characteristic Analysis for Factor 1}

The emotional word pairs such as "simple-gorgeous" and "plain - fashionable" have a greater correlation with the first factor. The "simple - gorgeous" and "plain - fashionable" emotional word pairs can explain the same factor, indicating that there is also a correlation between word pairs. According to the quantitative description of emotions in color psychology [5], warm colors and high color brightness will give a gorgeous visual impact. Color saturation, cool and warm and color contrast can represent the emotional characteristics of male T-shirt images, indicating that there is also a relationship with the emotional description of factor 1 . As shown in Table 1, the average saturation value, average tonal value, and color contrast value of the image:

Table 1. Saturation, hue and contrast values of males T-shirt images

\begin{tabular}{ccccc}
\hline Image & Factor 1 & $\begin{array}{c}\text { Average } \\
\text { Saturation }\end{array}$ & $\begin{array}{c}\text { Average } \\
\text { Saturation }\end{array}$ & Contrast \\
\hline Fig(a) & -2.0243 & 5.3535 & 328.1318 & 0.9870 \\
Fig(b) & -1.7720 & 3.3561 & 228.2734 & 1.1615 \\
Fig(c) & -0.3247 & 30.3509 & 196.4423 & 2.7501 \\
Fig(d) & -0.2215 & 87.3727 & 75.3659 & 5.7161 \\
Fig(e) & 0.3745 & 79.7302 & 74.1314 & 5.2451 \\
Fig(f) & 0.4570 & 73.8134 & 52.0021 & 5.1903 \\
\hline
\end{tabular}

It can be seen from Table 1 that the factor value, the average saturation value, and the contrast value of the image are large, and the average tonal value is small, otherwise the result is different. The male T-shirt image corresponding to the data in Table 1 is shown in Figure 1:
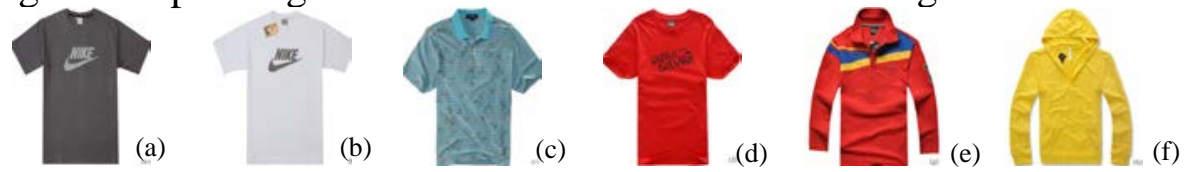

Figure 1. Male T-shirt Images of Table 1

As is known from TABLE I and Figure 1 , (a), (b) and (c) have smaller average saturation and color contrast values, and the average tonal value is larger; The result of (d), (e), (f) are reversed, indicating that the deeper the saturation, the brighter the color purity, the more vivid the image will be stylish of feeling. The numerical analysis in the table is consistent with the previous qualitative description of the emotion, so the image characteristics of the first factor are characterized by saturation-warm fuzzy histogram and color contrast.

\section{A Color Semantics Fuzzy Membership Functions}

In color psychology, qualitative description of color comes from human visual subjective perception, which has ambiguity, while fuzzy membership function is quantified by psychological perception. Usually, humans use language to describe things when they are ambiguous. Image recognition and retrieval is a computer simulation of human thinking processes to represent things information. This is fuzzy set concept [3].

A fuzzy set A on the domain $X$ means that any $x$ belongs to $X$, and there is a number $\mu_{A}(x)$ corresponding to 0 to 1 corresponding to it. So, there is a mapping:

$\mu_{A}: X \rightarrow[0,1] \quad x \rightarrow \mu_{A}(x)$

The linguistic description of the psychological quantities such as saturation, warmth and hue of color can calculate the membership function through the fuzzy set method. Such as, "Colorless", "very light", "medium thick", "medium thick", "darker-color", "very thick" of subordinate function language is used to describe saturation. As shown in the functional formula of equation (4): 


$$
\begin{aligned}
\mu_{A}(C)=\left\{\begin{array}{lll}
1 & C<7 \\
\frac{16}{9} & 7 \leq C \leq 16 \\
0 & C>17
\end{array} \quad \mu_{\text {light-colored }}(C)=\left\{\begin{array}{ll}
\frac{C-7}{9} & 7 \leq C<16 \\
\frac{27-C}{11} & 16 \leq C<27 \\
0 & \text { other }
\end{array} \mu_{\text {Darker }}(C)=\left\{\begin{array}{ll}
\frac{C-27}{13} & 27 \leq C<40 \\
\frac{59-C}{19} & 40 \leq C<59 \\
0 & \text { other }
\end{array} \quad \mu_{\text {Very-Thick }}(C)= \begin{cases}\frac{C-16}{11} & 16 \leq C<27 \\
\frac{40-C}{13} & 27 \leq C<40 \\
0 & \text { other } \\
1 & \text { other }\end{cases} \right.\right.\right.
\end{aligned}
$$

Cold and warm is the human body's feeling of temperature environment. In color psychology, color is related to the cold and warm perception of human vision, and the color also has the corresponding psychological feeling of warm and cold color. The cold and warm membership function formula is:

$$
\mu_{\text {warm }}(x)=\left\{\begin{array}{ll}
\cos (x) & 0^{\circ} \leq x<90^{\circ} \text { 或 } 270^{\circ} \leq x \leq 360^{\circ} \\
0 & \text { other }
\end{array} \quad \mu_{\text {cold }}(x)= \begin{cases}\cos \left(x-180^{\circ}\right) & 90^{\circ} \leq x<270^{\circ} \\
0 & \text { other }\end{cases}\right.
$$

In summary, "colorless warm color", "very light warm", "medium warm", "darker warm", "very-thick warm" and "colorless cold color", "very light cold", "medium cold", "darker cold", "very-thick cold" of fuzzy set are described factor 1. Defining the membership function of each image in 10 images belongs to the membership function of the 10 categories. When automatically calculating the low-level features of each factor of the image, the system will count the membership degree of each pixel of the image, and finally obtain the 10-dimensional saturation-warm and fuzzy histogram. After experimentation, the saturation-cold-warm fuzzy histogram can better describe the

\begin{tabular}{|c|c|c|c|c|c|c|c|c|c|c|c|}
\hline Images & $\begin{array}{c}\text { Colorless } \\
\text { Warm }\end{array}$ & $\begin{array}{l}\text { Light } \\
\text { Warm }\end{array}$ & $\begin{array}{c}\text { Medium } \\
\text { Warm }\end{array}$ & $\begin{array}{c}\text { Darker } \\
\text { Warmer }\end{array}$ & $\begin{array}{l}\text { Very- } \\
\text { Thick }\end{array}$ & Contrast & $\begin{array}{c}\text { Colorless } \\
\text { Warm }\end{array}$ & $\begin{array}{c}\text { Light } \\
\text { Warm }\end{array}$ & $\begin{array}{c}\text { Medium } \\
\text { Warm }\end{array}$ & $\begin{array}{c}\text { Darker } \\
\text { Warmer }\end{array}$ & $\begin{array}{l}\text { Very- } \\
\text { Thick }\end{array}$ \\
\hline (a) & 0.8697 & $\begin{array}{c}0.021 \\
2\end{array}$ & 0.0002 & 0.8912 & $\begin{array}{c}0.000 \\
0\end{array}$ & 0.0286 & 0.0008 & $\begin{array}{c}0.000 \\
0\end{array}$ & 0.0295 & 0.0000 & $\begin{array}{c}0.987 \\
0\end{array}$ \\
\hline (b) & 0.0135 & $\begin{array}{c}0.001 \\
3\end{array}$ & 0.0021 & 0.0201 & $\begin{array}{c}0.001 \\
2\end{array}$ & 0.5432 & 0.0002 & $\begin{array}{c}0.000 \\
0\end{array}$ & 0.5435 & 0.0000 & $\begin{array}{c}1.161 \\
5\end{array}$ \\
\hline (c) & 0.0879 & $\begin{array}{c}0.004 \\
9\end{array}$ & 0.0000 & 0.0930 & $\begin{array}{c}0.000 \\
0\end{array}$ & 0.1340 & 0.0946 & $\begin{array}{c}0.104 \\
9\end{array}$ & 0.5522 & 0.2588 & $\begin{array}{c}2.750 \\
1\end{array}$ \\
\hline (d) & 0.0003 & $\begin{array}{c}0.000 \\
0\end{array}$ & 0.0000 & 0.0003 & $\begin{array}{c}0.000 \\
0\end{array}$ & 0.0027 & 0.0016 & $\begin{array}{c}0.111 \\
4\end{array}$ & 0.1071 & 0.0044 & $\begin{array}{c}3.318 \\
8\end{array}$ \\
\hline (e) & 0.0200 & $\begin{array}{c}0.021 \\
2\end{array}$ & 0.0204 & 0.1017 & $\begin{array}{c}0.038 \\
6\end{array}$ & 0.0360 & 0.0312 & $\begin{array}{c}0.024 \\
9\end{array}$ & 0.7288 & 0.6375 & $\begin{array}{c}4.323 \\
4\end{array}$ \\
\hline (f) & 0.0047 & $\begin{array}{c}0.002 \\
2\end{array}$ & 0.0043 & 0.9868 & $\begin{array}{c}0.974 \\
5\end{array}$ & 0.0003 & 0.0003 & $\begin{array}{c}0.000 \\
4\end{array}$ & 0.0021 & 0.0009 & $\begin{array}{c}5.716 \\
1\end{array}$ \\
\hline (g) & 0.0083 & $\begin{array}{c}0.005 \\
1\end{array}$ & 0.0062 & 0.9275 & $\begin{array}{c}0.908 \\
2\end{array}$ & 0.0006 & 0.0004 & $\begin{array}{c}0.001 \\
0\end{array}$ & 0.0347 & 0.0326 & $\begin{array}{c}5.245 \\
1\end{array}$ \\
\hline (h) & 0.0217 & $\begin{array}{c}0.005 \\
7\end{array}$ & 0.0065 & 0.6509 & $\begin{array}{c}0.617 \\
5\end{array}$ & 0.0075 & 0.0001 & $\begin{array}{c}0.000 \\
0\end{array}$ & 0.0077 & 0.0000 & $\begin{array}{c}5.190 \\
3\end{array}$ \\
\hline
\end{tabular}
emotional semantics of the image.

Table 2. Saturation - cold and warm fuzzy histogram of male T-shirt images

It can be seen from Table 2 that the feature fuzzy membership description uses five, and the extracted saturation-cold-warm feature values are calculated in detail, which is more refined than the human-perceived color. The image in the table is shown in Fig. 1, (a) and (b) are black and white, which are achromatic, the data is mainly biased to be colorless, and the black and white pixel value density (saturation) is high, and the color contrast is small. From the overall tone, the images (c) in Figure 1 are biased toward richer-cold tones; (d), (e) and (f) are biased toward warm tones. It can be concluded that the characteristics of the male T-shirt image in terms of color saturation and cold and warm features can express the color change of the image, which is beneficial to the estimation of the emotion factor 1 . 
Through the above analysis, the 10-dimensional saturation-cold-warm histogram and color contrast are closely related to the emotional description of factor 1, and the 11-dimensional feature can be used as the lower-level feature corresponding to the factor 1 of the emotional factor space of the male T-shirt image.

\section{Characteristic Analysis for Factor 2}

Factor 2 is mainly related to the "classical - modern" and "calm - sportive" descriptors. From the perspective of color psychology theory, the emotional perception of such words is related to the richness and purity of the color of the image, and the contrast is high, the color is multicolored, and the vividness gives a feeling of leisure and movement.

The gray histogram can analyze the pixel distribution of the image and analyze the essential features of the image. The gray histogram is a function of the statistical value of the gray level distribution in the image according to the magnitude of the gray value and the frequency at which the pixel of the quantified image pixel is located. The formula for calculating the gray histogram [6] is:

$P\left(r_{k}\right)=n_{k} / N$

The abscissa of the gray histogram represents the gray level, generally indicated by $r$, and the ordinate indicates the number of occurrences of the gray level or the frequency $P\left(r_{k}\right)$ of the gray level pixel. In the formula, $\mathrm{N}$ is the total number of pixels of a male T-shirt image, $r_{k}$ is the number of pixels of the k gray level, $r_{k}$ is the k gray level, and $P\left(r_{k}\right)$ is the frequency at which the gray level pixels appear.

The grayscale histogram pixel distribution of each male T-shirt image as shown in Figure 2:

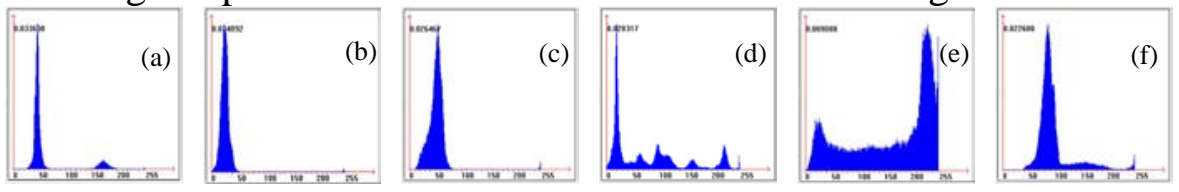

Figure 2. Grayscale Histogram of Male T-shirt Images

The corresponding male T-shirt image in Figure 2 is shown in Figure 3:
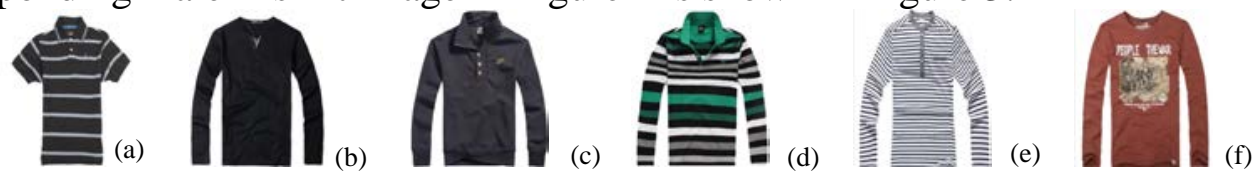

Figure 3. Sample of Male T-shirt Images

As shown in the above figure, the pixel distributions of (a), (b) and (c) in Figure. 2 are concentrated on a small number of grayscale values and narrow grayscale regions, and the grayscale values are larger than others. The maximum values of the gray values of (d), (e), (f) appear less and lower of contrast value, indicating that the color is relatively simple. In Fig. 2, the gray values of the (e) and (f) pixel distributions are more gray and the distribution is more evenly distributed, indicating that has multi-level color. It can be seen that the gray histogram plus color contrast can be characterized by a factor of two. In summary, the image can extract the grayscale features of the image, and after numerical normalization, the relationship between the low-level features of image and the factor 2 are described by the 256-dimensional gray histogram and the 1-dimensional color contrast.

\section{Characteristic Analysis for Factor 3}

Emotional words such as "gentle - strong" and "elegant - rugged" are related to factor 3. According to the theory of color psychology, the smoothness, contrast and images coarseness are closely related. It can be represented by the texture features of the image. Tamura [7-8-9], includes directionality, contrast, coarseness, line-likeness, regularity and roughness, is a texture feature extraction algorithm based on human visual perception. But the main components are direction, contrast and roughness. 
Table 3. Tamura Texture Feature Parameters and Average Hue of Images in Figures 4

\begin{tabular}{cccccc}
\hline Image & Factor 3 & Coarseness & Contrast & Directionality & Average Hue \\
\hline (a) & -0.4838 & 11.1993 & 14.4569 & 2193.9760 & 228.2734 \\
(b) & -0.1535 & 10.0967 & 50.2518 & 3536.8471 & 148.9236 \\
(c) & -0.6224 & 9.4460 & 32.8329 & 2913.3173 & 52.0021 \\
(d) & 0.7562 & 10.7815 & 78.1436 & 3495.1701 & 74.1314 \\
(e) & 0.4327 & 8.6159 & 95.5331 & 3023.2460 & 253.6596 \\
(f) & -0.3029 & 8.2527 & 88.3805 & 2458.1191 & 215.0706 \\
\hline
\end{tabular}

The images in Table 3 are shown in Figure 4. The results obtained in Table 3 are sorted, result is: coarseness: a, d, b, c, e, f; contrast: e, f, d, b, c, a; directionality: b, d, e, c, f, a.

It can be seen from the calculated values of the male T-shirt image samples selected in the table that the coarseness values of (a), (b), (c), and (d) are larger than (e), (f). The image (a) is larger than the roughness of the image sample (d), but the contrast of (d) is larger than (a), indicating that the image (d) is finer than (a) and the pixel distribution intensity is large. The image with a small coarseness value has a tight orientation, a well-ordered order, and a finer pixel distribution. The image gives a soft feeling. The average hue values of images (c) and (d) are small, and are warmer longs to $\left[0^{\circ}, 90^{\circ}\right.$; while other image samples in the above table have larger values, between $90^{\circ}$ to $270^{\circ}$, it is a cool color. The qualitative description is consistent with the visual perception of the image.
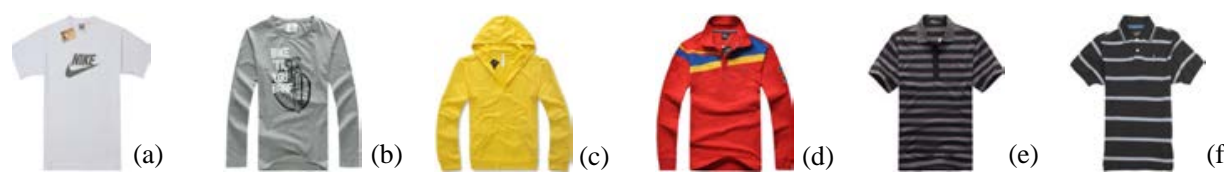

Figure 4. Male T-shirt Images in Table 3

In summary, from the qualitative and quantitative point of view, these characteristics are related to factor 3 , so we use Tamura texture feature extraction algorithm and average hue to analyze the texture of male T-shirt image samples

\section{Conclusion}

In this paper, factor 1 , factor 2 and factor 3 in the emotional factor space of male T-shirt images and corresponding lower-level features are analyzed. According to the analysis, factor 1 can be characterized by the low-level feature of "10-dimensional saturation-cold-warm fuzzy histogram plus 1-dimensional color contrast"; factor 2 can be characterized by "256-dimensional gray histogram and color contrast"; 3 can be characterized by a 3-dimensional Tamura texture extraction algorithm component parameter plus a 1-dimensional average hue. The analysis reveals the relationship between the emotional semantics of male T-shirts and low-level features, and also lays the foundation for the realization of emotional semantic recognition and retrieval of content-based male T-shirt images.

\section{Acknowledgments}

The work described in this article was supported by grants from the Science and Technology General project of Beijing Municipal Commission of Education (No.AJ2016-11).

\section{References}

[1] H. B. Zhang, T. J. Huang, Y. Xiu, et. Fabric image emotional semantic analysis based on color and texture features [J]. Journal of Tianjin Polytechnic University, 2013, 32(4):26-32(In Chinese).

[2] H. B. Zhang, T. J. Huang, R. P. Liu. Emotional semantic analysis of men's suit image based on color features [J]. Journal of Donghua University (Natural Science), 2013, 39(2):185-190,195(In Chinese). 
[3] W. N. Wang. Research on features extraction, retrieval and classification of image based on emotional semantic [D]. Guangzhou: South Chain University of Technology, 2005(In Chinese).

[4] H. B. Zhang, C. Y. Zhang. Study on the emotion factor space of men's T-shirt [C]. 2016 International Conference on Information Science and Control Engineering, Beijing, July, 2016(In Chinese).

[5] W. Li. Research and implementation of clothing recommendatio-n system based on emotional semantics [D]. Shanghai: Donghua University, 2013(In Chinese).

[6] X. R. Zhang, G. W. Lei. A background subtraction algorithms of object detection based on histogram [J]. 2010, (02): 45-47(In Chinese).

[7] J. F. Ning, L. Zhang, Z. David, et al. Robust object tracking using joint color-texture histogram[J]. International Journal of Pattern Recognition \& Artificial Intelligence, 2011, 23(7):1627-1639.

[8] H. Tamura, T. Yamawaki, S. J. Mori. Texture features corresponding to visual perception. IEEE SMC, 1978, 8(6):460-473.

[9] F. Y. Yuan, H. X. Wang, Z. Q. Yang. Research for texture analysis in content - based image retrieval [J]. New Technology of Library and Information Service, 2006(01):59-61(In Chinese). 